\title{
Modified analytical model for subthreshold current in short channel MOSFET"s
}

\author{
Mohammad Mahdi Khafaji ${ }^{a)}$, Mahmoud Kamarei ${ }^{\mathrm{b})}$, \\ and Behjat Forouzandeh ${ }^{\mathrm{c})}$ \\ University of Tehran, Tehran, Iran \\ a)mahdi_khafaji@yahoo.com \\ b) kamarei@ut.ac.ir \\ c)bforooz@ut.ac.ir
}

\begin{abstract}
In this paper an analytical drain current model in subthreshold region is proposed and can be used for MOS or PD-SOI transistors. The model can describe output current dependency on the drain voltage accurately as well as including short channel effects. The proposed model needs only two fitting parameters to predict the current of a transistor with very good accuracy and simply can be used in simulators. To validate the model, it is compared with two short channel MOS transistors with different technologies by HSPICE simulations.
\end{abstract}

Keywords: subthreshold, DIBL, MOS Modeling, PD-SOI

Classification: Electron devices

\section{References}

[1] B. C. Paul, A. Raychowdhury, and K. Roy, "Device optimization for digital subthreshold logic operation," IEEE Trans. Electron Devices, vol. 52, no. 2, pp. 237-247, Feb. 2005.

[2] A. Valentian, O. Thomas, A. Vladimirescu, and A. Amara, "Modeling subthreshold SOI logic for static timing analysis," IEEE Trans. VLSI Systems, vol. 12, no. 6, pp. 662-669, June 2004.

[3] B. Agrawal, V. K. De, J. M. Pimbley, and J. D. Meindl, "Short channel models and scaling limits of SOI and bulk MOSFETs," IEEE J. SolidState Circuits, vol. 29, no. 2, pp. 122-125, Feb. 1994.

[4] S. Baishya, A. Mallik, and C. K. Sarkar, "A subthreshold surface potential model for short-channel MOSFET taking into account the varying depth of channel depletion layer due to source and drain junctions," IEEE Trans. Electron Devices, vol. 53, no. 3, pp. 507-514, March 2006.

[5] T. Tsuchiya, Y. Sato, and M. Tomizawa, "Three mechanisms determining short-channel effects in fully-depleted SOI MOSFETs," IEEE Trans. Electron Devices, vol. 45, no. 5, pp. 1116-1121, May 1998.

[6] T. A. Fjeldly and M. Shur, "Threshold voltage modeling and the subthreshold regime of operation of short-channel MOSFETs," IEEE Trans. Electron Devices, , vol. 40, no. 1, pp. 137-145, Jan. 1993.

[7] A. A. Mutlu and M. Rahman, "Two-dimensional analytical model for drain induced barrier lowering (DIBL) in short channel MOSFETs," Proc. 


\section{Introduction}

Since the beginning of the integrated circuit era, the minimum feature size in CMOS technology has been reduced by more than two orders of magnitude to achieve improved performance, particularly in terms of speed of operation, dynamic power dissipation etc., in addition to increasing the packing density.

As the channel length is decreased, deviations from long channel behavior may occur. These departures, so called short-channel effects, arise as a consequence of two-dimensional potential distribution and high electric fields in the channel region [7]. Short-channel effects degrade the controllability of the gate voltage to drain current, which leads to the degradation of the subthreshold slope and the increase in drain off- or leakage current [5].

In the subthreshold regime in short-channel devices, a drain voltage induces lowering of the energy barrier between the source and the channel. This so-called Drain-Induced Barrier Lowering (DIBL) causes excess injection of charge carriers into the channel and gives rise to an increased subthreshold current. By introducing new CMOS technologies and shortening the channel length, this current is increased and thus should be modeled accurately.

Modeling of the leakage current is important for digital dynamic circuits as it determines the holding time of such circuits. In subthreshold regime devices have significantly higher gain because of the higher transconductanceto-current ratio [4]; thus the model would be important for designing of analog circuits too. Therefore, a simple and accurate analytical model is needed for hand estimation of circuit characteristics. Some models such as EKV and low-power transregional MOSFET have been developed for the design and simulation of low-voltage, low-current analog and mixed-signal circuits [2]. The disadvantage of these models is their relatively complex drain current equations. Other models, such as the physical Alpha-Power Law MOSFET have been developed in order to simplify the drain-current equations but the Alpha-Power Law MOSFET from which it is derived does not describe the subthreshold characteristic. The shortcoming of this model is its inadequate moderate-inversion formulation [2].

Also there are some models that describe the dependence of drain current to gate-source voltage with high accuracy but in large drain voltages, the change of drain current has not been considered adequately. In [2], e.g., without analytical explanation, a linear term is added to involve high voltage drain effects.

The objective of this paper is to develop an analytical model for the drain voltage dependency of subthreshold currents which can adequately describe submicrometer devices and, at the same time, is simple enough to be implemented in circuit simulators [6]. 


\section{Analytical subthreshold model}

Firstly, it is mentioned that in weak inversion regime two mechanisms are contributing in the making of current in the channel; thermionic emission and drift-diffusion [6]. But they are not exiting together, i.e. in some regions of channel one of them is dominant and in remaining places the other mechanism becomes major source of current. When the channel length is decreased, thermionic emission current will determine the main part of current. However, to have a precise model none of these mechanisms should be neglected.

In [6], by taking into account these two mechanisms and based on charge sharing model, a unified term for subthreshold current is derived. Having calculated drift-diffusion and thermionic emission currents, it is assumed that they act in serial manner. It means that because of the fact of different regions of functionality for these two mechanisms, series manner on the all channel length can be hypothesized. This is similar to a series combination of two admittances where the current is dominated by smaller of the two. Thus, the drain current will be dominated by the current-limiting mechanism which will be the one that corresponds to the highest impedance [6]. In line with this analogy, a unified expression for the subthreshold I-V characteristics was proposed as the following combination of the two currents:

$$
I_{D S} \approx\left[\frac{1}{I_{D S}(d d)}+\frac{1}{I_{D S}(t e)}\right]^{-1} \approx I_{0} \cdot f\left(V_{G S}\right) \cdot g\left(V_{D S}\right)
$$

In which

$$
\begin{gathered}
I_{D S}(t e)=k_{1} \cdot W \cdot \exp \left(\frac{\psi_{s}^{0}+V\left(x_{s}\right)}{V_{t h}}\right)\left[1-\exp \left(-\frac{V_{D S}}{V_{t h}}\right)\right] ; k_{1}=\frac{q N_{c} \delta D_{n} V_{0}}{\lambda V_{t h}} \\
I_{D S}(d d)=k_{2} \cdot W \cdot \frac{\exp \left(\frac{\psi_{s}^{0}+V\left(x_{s}\right)}{V_{t h}}\right)\left[1-\exp \left(-\frac{V_{D S}}{V_{t h}}\right)\right]}{1-\exp \left[-\frac{V_{0}\left(L-x_{s}-x_{d}\right)}{V_{t h} \lambda}\right]} ; k_{2}=\delta A^{*} T^{2} \quad(3)
\end{gathered}
$$

All parameters included in $k_{1}$ and $k_{2}$ are device parameters and depend on process and technology of transistor as well as temperature. It is clear from the above results that the dominant mechanism will be thermionic emission at small gate lengths and drift diffusion at larger gate lengths. However, due to complexity of this solution, physical insights into the dependence of short channel effects on the device parameters were masked and necessarily some simplifications are needed.

We remind here that the length of source-channel junction $\left(x_{s}\right)$ and drainchannel junction $\left(x_{d}\right)$ could be expressed as below [6].

$$
x_{s} \approx \sqrt{\frac{2 \varepsilon_{s}}{q N_{a}}\left(-\psi_{s}^{0}\right)} \quad, \quad x_{d} \approx \sqrt{\frac{2 \varepsilon_{s}}{q N_{a}}\left(V_{D S}-\psi_{s}^{0}\right)}
$$

According to above equations, following approximation is proposed:

$$
x_{d}-x_{s} \approx \alpha \cdot \sqrt{V_{D S}}
$$


Where $\alpha$ is a fitting parameter and is chosen near $\sqrt{\frac{2 \varepsilon_{s}}{q N_{a}}}$. It is noted that Eq. (5) is valid for relatively high drain voltages. By substituting Eq. (4) in Eq. (1) and doing some simplifications (shown in the Appendix), constitutive parts of drain channel are expressed by

$$
\begin{gathered}
I_{0}=k_{1} W \\
f\left(V_{G S}\right)=\exp \left(\frac{V_{G S}-V_{T}}{\eta V_{t h}}\right) ; \\
g\left(V_{D S}\right)=\left[1-\exp \left(-\frac{V_{D S}}{V_{t h}}\right)\right] \cdot \frac{k_{2} V_{t h} L-\alpha k_{2} V_{t h} \sqrt{V_{D S}}}{k_{1} V_{t h} L-\alpha k_{1} V_{t h \cdot} \sqrt{V_{D S}}+4 \chi d_{d e p}^{0} k_{2} V_{D S}}= \\
{\left[1-\exp \left(-\frac{V_{D S}}{V_{t h}}\right)\right] \cdot \frac{L-\alpha \sqrt{V_{D S}}}{k_{1} V_{t h} \cdot\left(L-\alpha \sqrt{V_{D S}}\right)+k_{3} k_{2} V_{D S}} ; k_{3}=4 \chi d_{d e p}^{0}}
\end{gathered}
$$

In comparison with I-V relation in [2], where the last expression of $g\left(V_{D S}\right)$, modeling DIBL effects, was replaced by a linear term without physical analysis, the proposed one not only has physical explanation but models strong DIBL effects on short channel devices.

By taking into account the floating body effect, the model could be generalized to include PD-SOI MOS transistors. The floating body of n-channel SOI transistors makes a positive charge during switching which results in an increase of the drain current or decrease in threshold voltage [2]. Proposed model in [2] involve this effect and the results are applicable here. Using same notation in [2] final model could be used for both bulk and SOI MOS transistors is expressed by:

$$
\begin{gathered}
I_{D S}=I_{0} \cdot f\left(V_{G S}\right) \cdot g\left(V_{D S}\right)=k_{1} \cdot W \cdot 10^{\left(\left|V_{G S}\right|-\left|V_{T}\right| / S\right)} \cdot g\left(V_{D S}\right) \\
V_{T}=V_{T 0}+\gamma\left(\sqrt{2 \cdot \psi_{F}-V_{B S} \cdot(1-\beta)}-\sqrt{2 \cdot \psi_{F}}\right) \\
S=\frac{K \cdot T}{q} \cdot \ln (10) \cdot\left(1+\frac{C_{D^{\prime}}}{C_{O X^{\prime}}}\right) \\
C_{D^{\prime}}=\sqrt{\frac{q \cdot N_{B} \cdot \varepsilon_{0} \cdot \varepsilon_{S i}}{2 \cdot\left(2 \cdot \psi_{F}-V_{B S}\right)}}
\end{gathered}
$$

In which $\gamma$ is the body factor, $\psi_{F}$ is the quasi-Fermi potential in the body, $C_{D^{\prime}}$ is the depletion-layer capacitance, $V_{B S}$ is the body to source voltage and function $g$ is the same as defined in Eq. (8). Thus complete model has two fitting parameters:
$\alpha$, to set drain voltage dependency,
$\beta$, that sets the saturation current.

The proposed analytical model presents another great advantage in SOI technology, for the ability to model drain current of a dynamic-threshold (DT) MOS transistors. By including the body effect in the $I_{D S}$ expression, we can easily model a DTMOS by substituting $V_{G S}$ for $V_{B S}$ in Eq. (9) to Eq. (12) [2]. 


\section{Simulation results}

After completing the description of the model, it is compared with two MOS models by HSPICE simulator. The first model is IBM $0.13 \mathrm{u}$ industrial model and the second is BSIM4.5 Berkeley SPICE model with $90 \mathrm{~nm}$ channel length. Simulations are done with various gate voltages and up to high drain voltages to maximize DIBL effect and test the ability of proposed model in this condition. As can be seen on the Fig. 1, there is good agreement between proposed model and SPICE simulations.



(a)

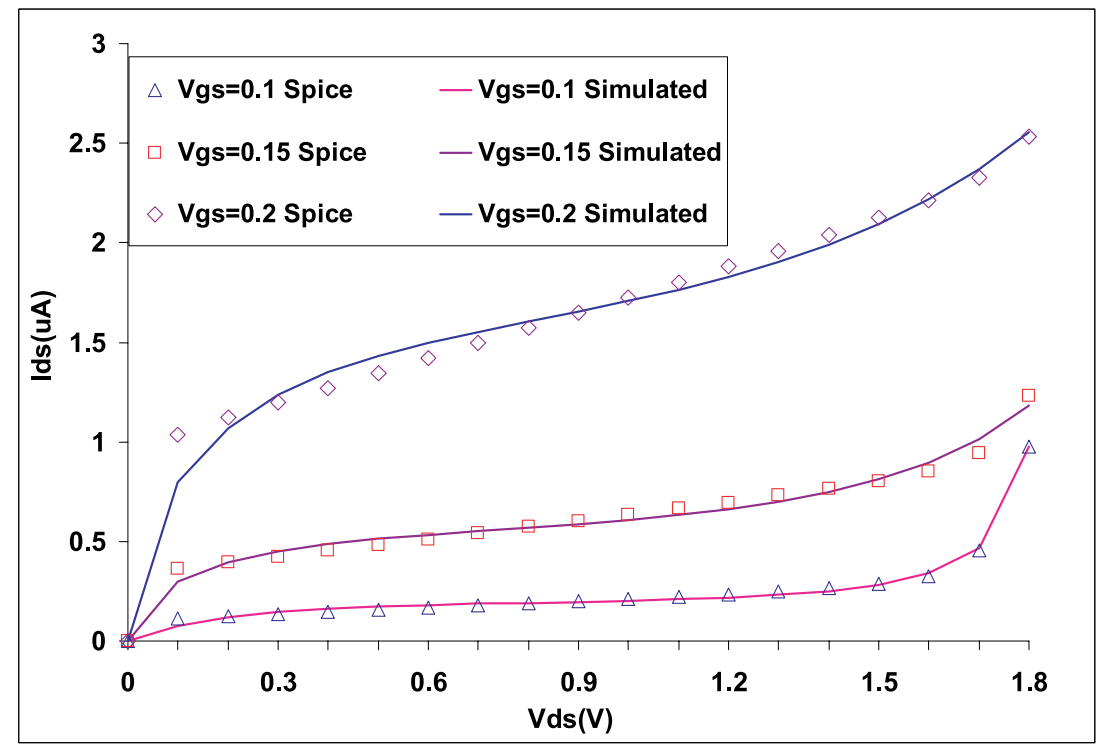

(b)

Fig. 1. Verification of proposed analytical model against SPICE simulations for (a) industrial IBM $0.13 \mathrm{u}$ model and (b) BSIM4.5 $90 \mathrm{~nm}$ Berkeley SPICE model. 


\section{Conclusion}

A simple analytical subthreshold model for the estimation of the performance of bulk CMOS transistors for digital and analog circuit designing has been presented. The dependence of the drain current on drain voltage (DIBL effects) in subthreshold region is included in the model. By adding the effect of body voltage the model easily is applicable for SOI CMOS circuits, since the body potential is especially important in SOI CMOS circuits. The model just needs two parameters to predict the drain current of a transistor with very good accuracy. This model has been compared with two short channel MOS transistors with different technologies by HSPICE simulations. The calculated characteristics are in very good agreement with simulated behavior of a CMOS transistor in industrial IBM $0.13 \mathrm{~m}$ and BSIM4.5 $90 \mathrm{~nm}$ processes.

\section{Appendix}

In this section detailed calculation for derivation of Eq. (8) will be demonstrated. By substituting Eq. (2) and Eq. (3) in Eq. (1), it can be rewritten

$$
\begin{aligned}
& I_{D S}=k_{1} W \cdot \exp \left(\frac{\psi_{s}^{0}+V\left(x_{s}\right)}{V_{t h}}\right)\left[1-\exp \left(-\frac{V_{D S}}{V_{t h}}\right)\right] \times \\
& {\left[1+\frac{k_{1}}{k_{2}}-\exp \left(-\frac{V_{0}\left(L-x_{s}-x_{d}\right)}{\lambda V_{t h}}\right)\right]^{-1}}
\end{aligned}
$$

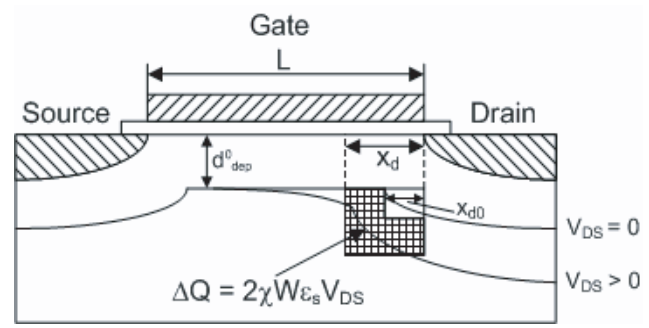

Fig. 2. Simplified model of the drain-bias-induced charge $\Delta \mathrm{Q}$ in the substrate under the gate according to the principle of charge sharing.
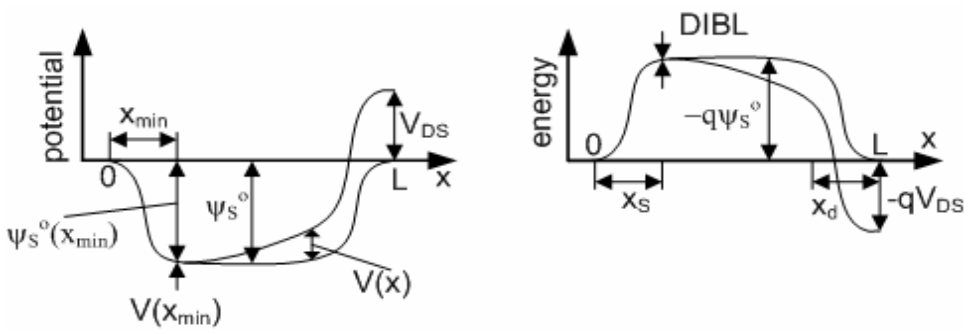

Fig. 3. Band Diagram (left) and potential profile (right) at the semiconductor-insulator interface of an nchannel MOSFET. The symmetrical profiles correspond to $\mathrm{V}_{\mathrm{DS}}=0$, and the asymmetrical profiles to $\mathrm{V}_{\mathrm{DS}}>0$. Figure indicates the origin of the Drain-Induced Barrier Lowering (DIBL). 
Regarding Fig. 2 and Fig. 3 below equations are transcribed from [6]:

$$
V_{0}=\frac{2 \chi V_{D S} d_{d e p}^{0}}{\lambda} \cdot\left[\cosh \left(\frac{L-x_{d}}{\lambda}\right)-\cosh \left(\frac{x_{s}}{\lambda}\right)\right]^{-1}, \quad \frac{\psi_{S}^{0}}{V_{t h}}=\frac{V_{G S}-V_{T}}{\eta V_{t h}} .
$$

And with first order simplification:

$$
\begin{gathered}
V_{0} \approx \frac{4 \chi V_{D S} d_{d e p}^{0} \lambda}{\left(L-x_{d}-x_{s}\right)\left(L-x_{d}+x_{s}\right)} \quad, \quad V\left(x_{s}\right) \approx 0 \\
I_{D S} \approx k_{1} W \cdot \exp \left(\frac{V_{G S}-V_{T}}{\eta V_{t h}}\right)\left[1-\exp \left(-\frac{V_{D S}}{V_{t h}}\right)\right] \times\left[\frac{k_{1}}{k_{2}}+\frac{4 \chi V_{D S} d_{d e p}^{0}}{V_{t h}\left(L-x d+x_{s}\right)}\right]^{-1}
\end{gathered}
$$

By some algebra calculations achieving final equation is straightforward.

\section{Acknowledgments}

The authors would like to thank Center of Excellence on Applied Electromagnetic Systems, University of Tehran, and Iran Telecommunication Research Center (ITRC) for financial support. Also, the authors thank Abo-Alfadhl Al-Abbas for his helping during all this work. 